\title{
THE TIGGE PROJECT AND ITS ACHIEVEMENTS
}

by Richard Swinbank, Masayuki Kyouda, Piers Buchanan, Lizzie Froude, Thomas M. Hamill,

Tim D. Hewson, Julia H. Keller, Mio Matsueda, John Methren, Florian Pappenberger, Michael Scheuerer, Helen A. Titley, Laurence Wilson, and Munehiko Yamaguchi

The rich dataset of ensemble predictions from the TIGGE project has supported a wide range of scientific studies and new products for forecasting severe weather

T he Observing System Research and Predictability Experiment (THORPEX) was a decade-long international research and development program to accelerate improvements in the accuracy

AfFILIATIONS: SWINBANK, BUCHANAN, AND TITLEY-Met Office, Exeter, United Kingdom; KYOuDA-Japan Meteorological Agency, Tokyo, Japan; Froude AND Methren-University of Reading, Reading, United Kingdom; HamiLl AND Scheuerer-Physical Sciences Division, NOAA/ESRL, Boulder, Colorado; HeWSON-European Centre for Medium-Range Weather Forecasts, Reading, United Kingdom; KelleR-Deutscher Wetterdienst, Offenbach, Germany; Matsueda-University of Tsukuba, Tsukuba, Ibaraki, Japan, and University of Oxford, Oxford, United Kingdom; PAPPENBERGEREuropean Centre for Medium-Range Weather Forecasts, Reading, and University of Bristol, Bristol, United Kingdom, and Hohai University, Nanjing, China; WILSON-Environment Canada, Montreal, Quebec, Canada; YAMAGUCHI-Meteorological Research Institute, Tsukuba, Ibaraki, Japan

CORRESPONDING AUTHOR: Richard Swinbank, Met Office, FitzRoy Road, Exeter EXI 3PB, United Kingdom

E-mail: richard.swinbank@metoffice.gov.uk

The abstract for this article can be found in this issue, following the table of contents.

DOI:10.1175/BAMS-D-13-00191.I

A supplement to this article is available online (10.1175/BAMS-D-13-00191.2)

In final form 23 February 2015

○2016 American Meteorological Society and benefits of high-impact weather forecasts up to two weeks ahead (WMO 2005; Shapiro and Thorpe 2004a,b). THORPEX was established in 2003 as part of the World Meteorological Organization (WMO) World Weather Research Program (WWRP). The execution phase of THORPEX lasted a decade, from 2005 to 2014. It is thus an opportune time to take stock of the achievements of the THORPEX program and its component parts. This paper is focused on achievements related to The International Grand Global Ensemble (TIGGE) project, while subsequent articles will cover the broader achievements of THORPEX.

TIGGE was established to support a range of THORPEX research activities by providing operational ensemble forecast data to the international research community. The original name of TIGGE, “THORPEX Interactive Grand Global Ensemble," reflected the THORPEX vision for the development of a future global interactive forecast system (GIFS), including the use of ensemble prediction systems (EPSs) that would be configured interactively in response to varying weather situations and user needs.

The THORPEX program covered three major research areas: predictability and dynamical processes, data assimilation and observing systems, and ensemble forecasting. These correspond to the three WWRP-THORPEX working groups: predictability 
and dynamical processes (PDP), data assimilation and observing system (DAOS), and GIFS-TIGGE. TIGGE provides forecast data to support all three of these research strands. Although on-demand ensemble predictions are not yet a routine operational reality, there has been an increasing use of techniques to combine ensemble predictions for operational forecast products. TIGGE has proved particularly valuable as a dataset to support research on predictability and dynamical processes and the development of ensemble-based forecast products. By making ensemble prediction data from leading operational forecast centers readily available for research, TIGGE has enhanced cooperation between the academic and operational meteorological communities. Because of the value of the data, it has been agreed that TIGGE will be continued for at least a further 5 yr. A new name, "The International Grand Global Ensemble," has been adopted to reflect the completion of THORPEX, while retaining the TIGGE acronym.

The TIGGE database contains ensemble predictions from 10 global numerical weather prediction (NWP) centers and is available via three archive centers: ECMWF, NCAR (until 2015), and CMA (see Table 1 for a list of TIGGE partners and their acronyms, as used in this article, and see the sidebar on "Accessing TIGGE data" for information on accessing the data). Since the basis of TIGGE is to support research, and not operations, the technical setup (Worley et al. 2008) is not designed to support real-time exchange of data. Instead, the data are made available to users $48 \mathrm{~h}$ after the initial time of each forecast. A TIGGE-Limited Area Model (LAM) panel was also established to apply TIGGE concepts to limited area model ensembles. Several European regional ensembles are now available from a TIGGE-LAM archive established at ECMWF during 2014.

Because of the huge data volume, it was not feasible to include a full range of model fields at all levels in the TIGGE database; instead fields were selected taking into account user requirements discussed at a workshop hosted by ECMWF (Richardson et al. 2005). Documentation of the archived fields is available on the TIGGE project website (http://tigge .ecmwf.int) and in Bougeault et al. (2010). The TIGGE data are stored in gridded binary (GRIB2) format, the standard established by WMO for the storage of gridded binary data that was designed to cater for ensembles. The TIGGE partners agreed upon a series of standards and conventions to enable users to read forecast data from any of the TIGGE partners using the same computer code. The TIGGE data portals include links to tools contributed by TIGGE users, which are designed to help new users read and plot the TIGGE data, including tools to convert the GRIB2 data to NetCDF format if required.

Since it was launched on 1 October 2006, the usage of the TIGGE archive has increased steadily. During calendar year 2013, there were at least 110 active users of the archive each month, and about $800 \mathrm{~TB}$ of data were accessed from the database over the year.

Bougeault et al. (2010) described some early results from TIGGE and pointed out that multimodel grand ensemble systems - combining predictions from several TIGGE models-have been demonstrated to give additional skill for some types of forecast parameters. The section on the "Verification, combination, and calibration of TIGGE forecasts" of this paper reviews the result of recent research on that topic plus other studies evaluating the quality of the TIGGE forecasts.

TABLE I. TIGGE project partners.

\begin{tabular}{|lll|}
\hline Center & Country & Acronym \\
\hline Bureau of Meteorology & Australia & BoM \\
\hline China Meteorological Administration & China & CMA \\
\hline Canadian Meteorological Centre & Canada & CMC \\
\hline Centro de Previsão de Tempo e Estudos Climáticos & Brazil & CTPEC \\
\hline European Centre for Medium-Range Weather Forecasts & Europe & ECMWF \\
\hline Japan Meteorological Agency & Japan & JMA \\
\hline Korea Meteorological Administration & Korea & KMA \\
\hline Météo-France & France & MF \\
\hline Met Office & United Kingdom & UKMO \\
\hline National Center for Atmospheric Research & United States & NCAR \\
\hline National Centers for Environmental Prediction & United States & NCEP \\
\hline National Climatic Data Center & United States & NCDC \\
\hline
\end{tabular}




\section{ACCESSING TIGGE DATA}

n overview of TIGGE, with links to further

Ainformation and documentation, is given online (http:// tigge.ecmwf.int/).

The TIGGE data are available from the following web portals:

- ECMWF (http://apps.ecmwf.int/datasets/);

- CMA (http://wisportal.cma.gov.cn/wis/); and

- NCAR (http://rda.ucar.edu/; data up to the end of 2014 , available until the end of 2015).

The TIGGE-LAM archive enables researchers to have access to forecasts from several European regional EPSs. The forecasts are produced at high resolution (between $12-\mathrm{km}$ and 2-km grid spacing) and provide detailed forecasts up to a few days ahead. TIGGE-LAM data are available via the ECMWF portal shown above.

TIGGE has opened up the opportunity for researchers to use the ensemble data for a wide range of studies, particularly on predictability and dynamical processes. At the time of writing, around 120 TIGGErelated papers have been published. Highlights of studies of dynamics and predictability of both midlatitude and tropical systems are presented in the section on "Dynamics and predictability." A wide range of information about TIGGE is displayed on the TIGGE Museum website (see the sidebar on "The TIGGE Museum"), and several examples of graphical products from the website are used to illustrate this article.

Despite the fact that the TIGGE database was not designed to cater for real-time use, the section on "Applications for the forecast user community" shows that TIGGE has proved invaluable for the development of products to support forecasts and warnings of high-impact weather as part of the vision for GIFS. The final section of the paper looks beyond the THORPEX program and explores how the achievements of TIGGE should be built on in the future.

\section{VERIFICATION, COMBINATION, AND CALIBRATION OF TIGGE FORECASTS.}

Verification. The TIGGE database is designed to facilitate comparative verification of the ensembles contained therein, and many examples have been published. Figure 1, from the TIGGE Museum website, compares root-mean-square (RMS) errors of 500-hPa geopotential height for the Northern Hemisphere in winter 2013/14. The relative ordering of skill is typical of many other cases; ECMWF has lower errors than other centers, with tight competition for second place. Hamill (2012) found a somewhat similar relative ordering for precipitation over the contiguous United States. In a more extreme case of error differences, Hagedorn et al. (2012, their Fig. 3) showed that, in 2008/09, the 2-m temperature forecasts at the 1-week range from the ECMWF system were similar in quality to several of the least skillful forecast systems at the 1-day range. Of course, each system has been upgraded during the course of TIGGE, so these results will not necessarily reflect the precise relative or absolute performance of these systems at the current time.

Though it is preferable when available to verify against observations, analyses are often used instead to provide information on forecast quality that includes observation-sparse areas. Unfortunately, the relative performance of various modeling systems can depend strongly on which analysis is used for verification. For example, for low-level tropical regions, the model whose analysis was used as the verification field appeared to be the most accurate (see Park et al. 2008, their Fig. 14). The yearly mean, analyzed, 2-m temperature from five of the TIGGE systems was shown to vary by almost $5 \mathrm{~K}$ between the warmest and coldest analyses for a location in the Amazon basin (Fig. 2). Large differences were also commonplace for some upper-air variables and for data at other locations. Given these differences, verifying against more than one analysis is preferable because if a given model is unambiguously higher in skill than another regardless of which analysis was used, this lends credence to the result. Alternatively, a consensus of the more skillful analyses might be used.

\section{THE TIGGE MUSEUM}

The TIGGE Museum website was established by Mio Matsueda, with Tetsuo Nakazawa. The website is currently hosted by the University of Tsukuba (at http:// gpvjma.ccs.hpcc.jp/TIGGE/). It displays a variety of graphical information based on the TIGGE dataset, including

- statistical verification of TIGGE forecasts;

- ensemble-based forecasts of severe weather;

- forecasts of the Madden-Julian oscillation and blocking; and

- sample scripts to show how to download and plot TIGGE data.

The TIGGE Museum products are regularly updated with a 2-3-day delay and are available for noncommercial use. 
TIGGE medium-range ensemble forecasts

Z500 RMSE (Northern Hemisphere, DJF2013/14)

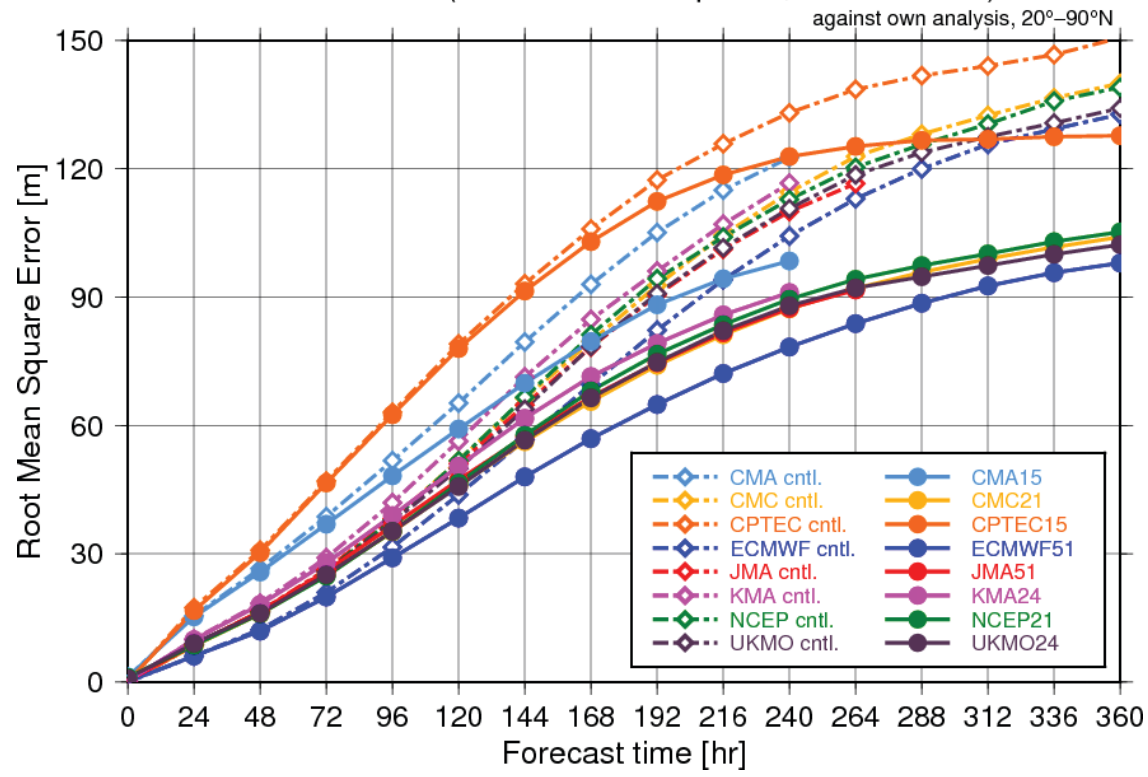

FIG. I. Comparison of the skill of Northern Hemisphere 500-hPa forecasts from systems contributing to TIGGE for Dec 2013 through Feb 20I4. Each forecast is verified against its own analysis. Solid lines show the RMS error of the ensemble mean, and dashed lines show the control member of each ensemble. Refer to Table I for forecast center abbreviations. The number following the center name indicates the number of ensemble members used.

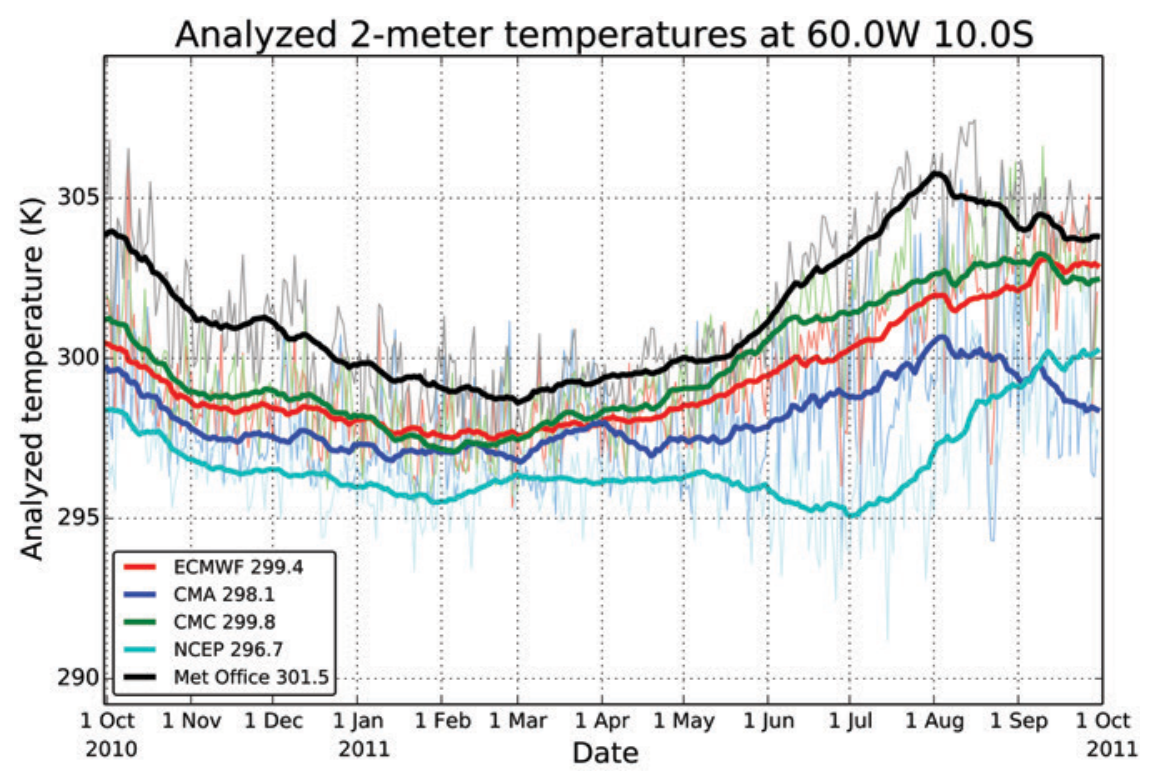

FIG. 2. Time series of daily (0000 UTC) 2-m temperature analyses from four different TIGGE analyses, here for a grid point in the Amazon basin $\left(10^{\circ} \mathrm{S}\right.$, $\left.60^{\circ} \mathrm{W}\right)$. The numbers associated with the legend indicate the yearly mean analyzed temperature. Thin, lighter-colored lines provide the daily analyses, and thicker, darker-colored lines provide the smoothed analysis, an average of \pm 15 days.
Combination. Probabilistic forecast skill and reliability can be improved through the combination of TIGGE data, that is, the generation of a multimodel grand ensemble by combining raw ensemble predictions from multiple centers. As mentioned in Hagedorn et al. (2005), "the key to the success of the multimodel concept lies in combining independent and skillful models, each with its own strengths and weaknesses." Two underlying assumptions behind the success of combination are that 1 ) the modeling systems may have independent (or nearly so) systematic errors, thus providing some benefit through cancellation, and 2) the modeling systems collectively may provide more realistic estimates of event probabilities than individually. Several studies have demonstrated such improvement, including Matsueda and Tanaka (2008), Park et al. (2008), Johnson and Swinbank (2009), Candille (2009), Hagedorn et al. (2012), and Hamill (2012). Large benefits have been found for quantities relevant to weather impacts such as surface air temperature, surface wind, and precipitation. Hamill (2012) showed that multimodel combination improved the overall skill and reliability of precipitation forecasts over the contiguous United States; similar results are shown in Fig. 3. There are both practical and theoretical considerations that 
Reliability, Day +3 $1.0 \mathrm{~mm}$

(a) ECMWF

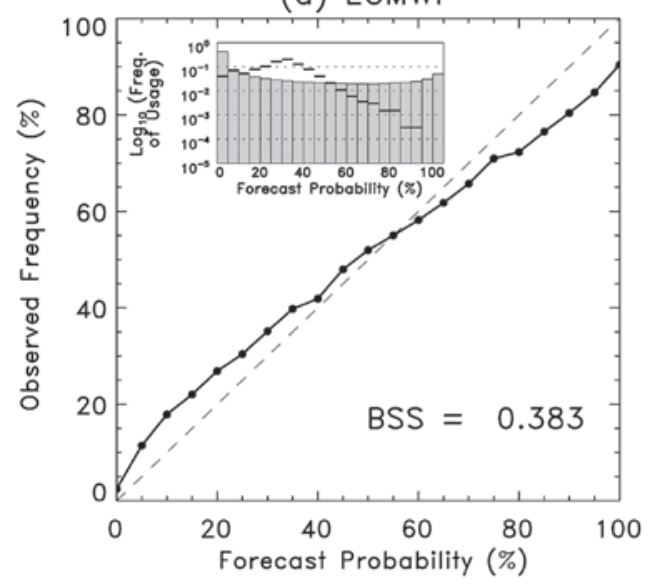

(c) $\mathrm{CMC}$

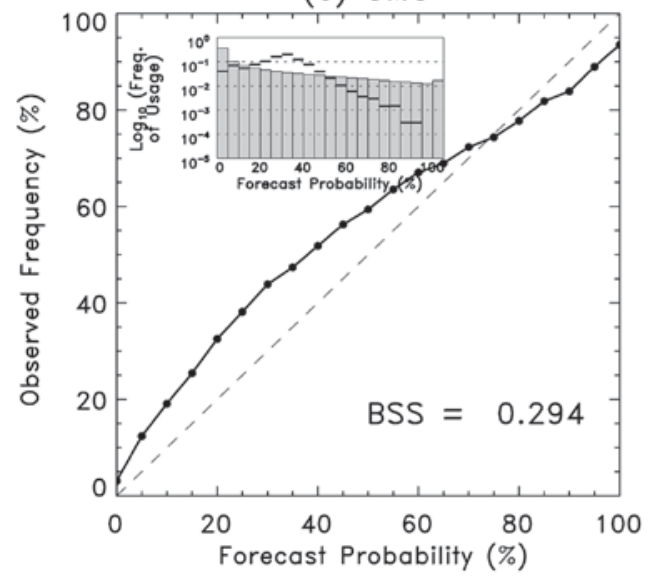

(b) NCEP

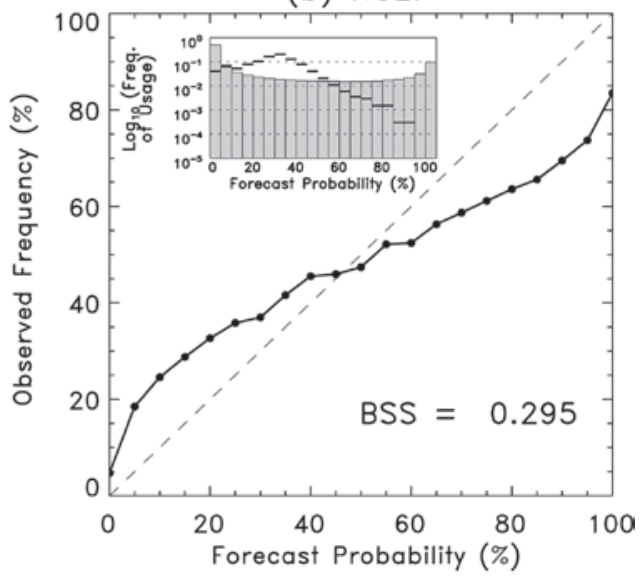

(d) UK Met Office

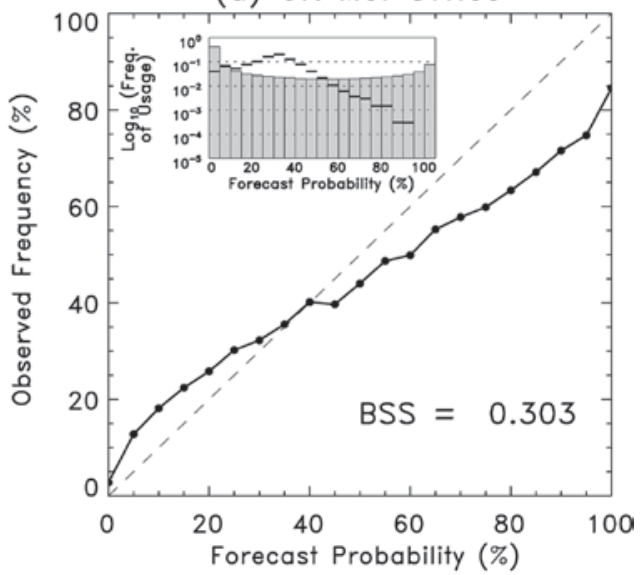

(e) Multi-model combination

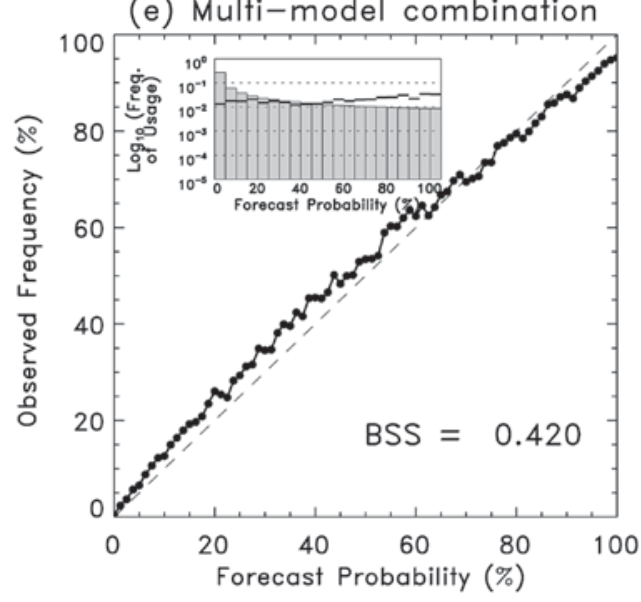

Fig. 3. Reliability diagrams for $T+48$ to $T+72 \mathrm{~h}$ accumulated precipitation forecasts on a $1^{\circ}$ grid over the contiguous United States, for (a)-(d) individual EPSs and for the (e) multimodel ensemble. This used the Jul-Oct $201 \mathrm{l}$ ensemble dataset as in Hamill (2012), but here the reliability diagrams were populated with forecasts from both 0000 and 1200 UTC initial conditions. Brier skill score (BSS) computations were performed as in Wilks (2006). The inset histogram shows the frequency with which forecasts were issued; horizontal solid lines therein denote the frequency distribution of climatological forecasts. 
will affect how much benefit users derive from multimodel ensemble combination. Practically, the global EPSs in TIGGE contain forecasts with differing qualities. Hagedorn et al. (2012) showed that the combination of the four highest-performing ensembles led to forecasts that were statistically significantly better than the raw ensemble guidance from the best-performing system. However, when the combination included data from all available TIGGE systems, there was no unambiguous statistical advantage, showing that some account needs to be taken of relative quality. More theoretically, as EPSs are upgraded (e.g., higher resolution, improved initialization procedures, and improved parameterizations), the systematic biases in each center's mean forecast will decrease. Should the prediction systems also incorporate more sophisticated methods for simulating the model uncertainty, their spread will also become more consistent with the ensemblemean error, as expected by theory. In this (desirable) situation, the simple combination of ensemble prediction data would become less beneficial, aside from the reduction in sampling error from the use of a larger ensemble. There will also be less benefit of combining data from current-generation EPSs if the combination is attempted after some postprocessing to remove bias and calibrate spread. The supplemental information (available online at http://dx.doi .org/I0.II75/BAMS-D-|3-00191.2) provides further discussion of how ensembles might be combined in the presence of correlated errors.

Calibration. Whether in combination or alone, the information provided by EPSs often requires some statistical postprocessing to reduce systematic errors as well as to deal with sampling error. TIGGE has provided a rich set of data that have enabled research on a range of potential methods for the calibration of ensemble predictions, contributing to the large body of literature on the subject (see, e.g., Jolliffe and Stephenson 2011). Which approach works best often depends on the variable in question; a postprocessing method that works well with temperature is probably not optimal for precipitation because of the different characteristics of their probability distributions.

The accuracy and reliability of postprocessed guidance may depend on the amount of training data available, particularly for more uncommon, highimpact events such as heavy precipitation. How does one obtain a sufficiently large sample when forecast models are updated every year or so, which may change the model's error characteristics? An ideal method is to use a reforecast dataset, incorporating a large number of forecasts of past cases that have been rerun with the current NWP system. The advantage of using training samples from a reforecast dataset for the calibration of surface temperature data is clearly shown in Hagedorn et al. (2012), although the results for precipitation from Hamill (2012) are less clear cut. Ideally the retrospective forecasts will have the same error characteristics as the operational model. Should the forecast modeling system change significantly, a new reforecast dataset should be generated. Because of the computational expense, many centers seek to provide statistically adjusted guidance using shorter training datasets, such as the 30-day training period used in part for the calibration in Hagedorn et al. (2012) and the 40-day training period used in Wilson et al. (2007). Shorter training periods have been shown to produce acceptable results for shorter-range forecasts of variables such as surface temperature, but larger sample sizes are increasingly valuable for longer-range forecasts and for forecasts of more rare events such as heavy precipitation.

\section{DYNAMICS AND PREDICTABILITY.}

Extratropical cyclones and storm tracks. Extratropical cyclones, and the associated baroclinic waves, are the primary cause of variability in weather across the midlatitudes. Mesoscale features embedded within cyclones, such as fronts, can bring both damaging surface winds and heavy precipitation leading to impacts such as widespread flooding.

The regions where extratropical cyclones frequently occur are often called storm tracks; the most prominent storm tracks in the Northern Hemisphere span the Atlantic and Pacific (PA) Oceans. The heat and moisture fluxes associated with cyclones dominate the poleward transport of energy in the atmosphere and therefore have a crucial influence on climate. Using TIGGE data, individual cyclones were tracked and systematic errors were diagnosed for the global ensemble forecasts from the 10 centers (Froude 2010, 2011; Fig. 4). This methodology has revealed valuable information about the representation of cyclones in numerical weather prediction models and their lower-resolution cousins-climate models. The ECMWF ensemble was found to have the highest level of performance in predicting cyclone position, intensity, and propagation speed. However, there may be some bias as all the ensembles were verified against the ECMWF analysis (as discussed in the section on "Verification"). Figure 4a also shows that the intensity of the cyclones was not predicted as well by the ensembles with lower spatial resolutions (NCEP, BoM, and CPTEC), perhaps indicating 

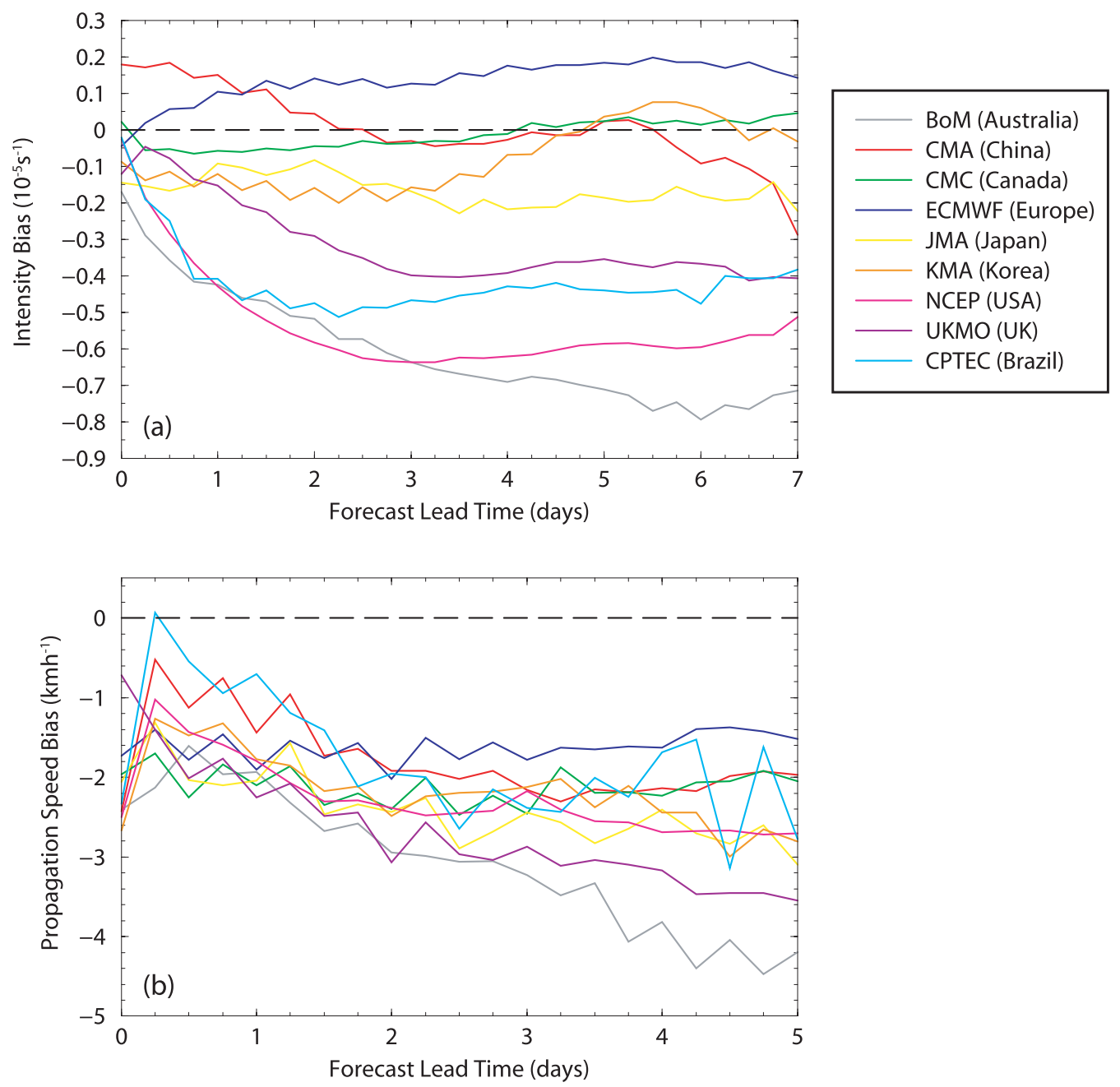

Fıg. 4. Bias in (a) intensity and (b) propagation speed of extratropical cyclones tracked in forecasts from the different global centers contributing to TIGGE, as a function of lead time (Froude 20I0).

some systematic errors in simulating the contraction and intensification of ascent into narrow regions as a result of latent heat release. An intriguing, but as yet unexplained, forecast error is that all the EPSs were found to underpredict the propagation speed of cyclones (Fig. 4b). Froude (2011) also assessed this bias in the ECMWF high-resolution forecast, and the bias was found to be significantly smaller than the lower-resolution EPS.

Ensembles of cyclone tracks can be displayed to illustrate uncertainty. This is illustrated for $T+72 \mathrm{~h}$ forecasts in Fig. 5 for the high-impact St. Jude's storm case on 28 October 2013 (see also Hewson et al. 2014). This intense cyclone caused a trail of severe damage across highly populated areas including southeast England, the Netherlands, and Denmark. Both the Met Office and ECMWF run the Hewson and Titley (2010) cyclonic feature identification and tracking methodology on their global ensembles, and the results are used by operational forecasters. The cyclonic features are detected using a combination of vorticity maxima and pressure minima. In Figs. $5 \mathrm{a}$ and $5 \mathrm{~b}$, the dots locate the centers of cyclonic features with intensities indicated by the colors. The scatter provides an immediate visual impression of the uncertainty in feature locations represented by the ensembles. The analyzed storm center reached Denmark at about 1400 UTC. Approximately half the Met Office ensemble clustered toward Denmark, the other solutions showing the cyclone nearer the United Kingdom. In contrast, all members of the ECMWF ensemble predicted the cyclone moving too slowly. The feature points and associated values were used to create forecasts of strike probability in Figs. $5 \mathrm{c}$ and $5 \mathrm{~d}$. They are somewhat analogous to the "cone of uncertainty" plots employed in hurricane forecasts (e.g., Majumdar and Finocchio 2010). The marked difference between the ECMWF 

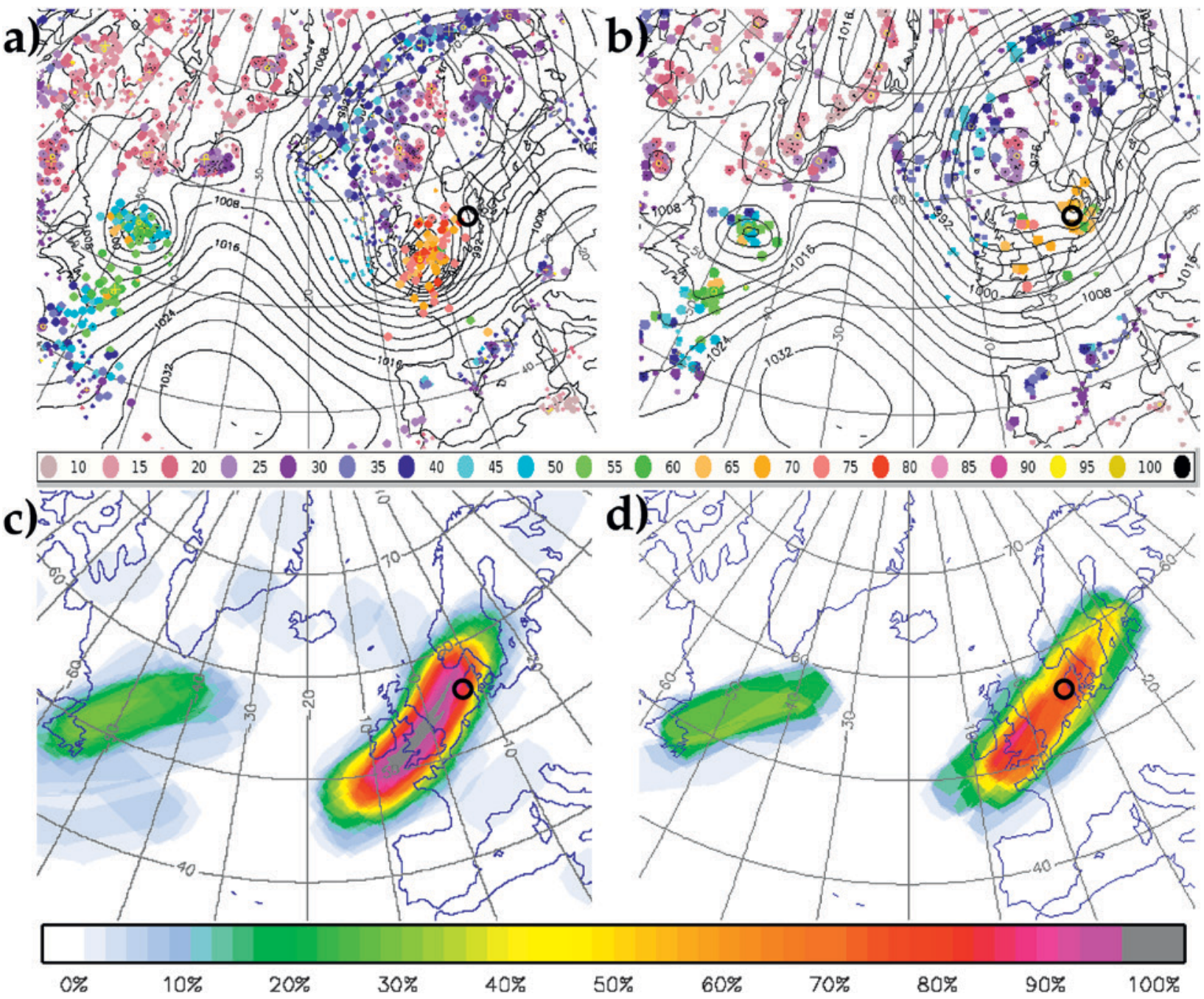

FIG. 5. Ensemble forecasts for a high-impact extratropical cyclone crossing the United Kingdom and Denmark. The circle shows the observed location of the cyclone at I200 UTC 28 Oct 20 I3. (top) “Dalmatian plots" representing cyclonic features in the $T+72 \mathrm{~h}$ ensemble forecast from (a) ECMWF and (b) Met Office. The features are colored by maximum wind speed (see scale in knots) within a $300-\mathbf{k m}$ radius at I-km altitude. Note that the features from every ensemble member are overlain, so the location of the cyclone is indicated by $\mathbf{5 2}$ or $\mathbf{2 4}$ dots for ECMWF and Met Office forecasts, respectively. Mean sea level pressure from the control run is also shown for both centers. (bottom) Cyclonic feature strike probability estimated from (c) ECMWF and (d) Met Office ensemble forecasts $(T+72 \mathrm{~h})$ using cyclonic feature tracking. At each point the color represents the probability that a moving cyclonic center associated with wind speeds over $60 \mathrm{kt}\left(\mathrm{I} \mathrm{kt}=0.5 \mathrm{I} 44 \mathrm{~m} \mathrm{~s} \mathrm{~s}^{-1}\right.$; at I-km altitude, within $300 \mathrm{~km}$ of the center) will at some point, within a centered 24-h window, be less than $300 \mathrm{~km}$ away.

and Met Office probability forecasts illustrates that ensemble forecasting systems are not perfect and more research is required to transform ensemble predictions into accurate probability forecasts for weather events.

Jet stream variability: Large-scale flow regimes and blocking. TIGGE has facilitated studies of large-scale, low-frequency variations in the jet stream. The jet stream is characterized by very large-scale meanders and the phenomenon of Rossby wave breaking.
Low-frequency variability is dominated by a few large-scale patterns [e.g., Cassou et al. (2004) identified four in the Euro-Atlantic (EA) sector]. It might be anticipated that low-resolution models would be able to simulate such patterns. However, Dawson et al. (2012) showed that lower-resolution (T159) simulations fail to capture the observed variability, while the free-running ECMWF model at the resolution of the ECMWF EPS (T511) captures the structure and variance of the large-scale patterns over the Atlantic. Doubling the resolution again to T1279 
obtains similar results, indicating convergence in the ECMWF model representation of low-frequency dynamics.

The TIGGE database was used by Matsueda (2009) to show that ensemble forecasts perform well in simulating the frequencies of EA and PA blocking, even after a lead time of 9 days. However, probabilistic forecasts of blocking over the PA sector were more skillful than those for the EA sector. Frame et al. $(2011,2013)$ took a different approach in quantifying the skill in the prediction of the probability of transition between three states of the North Atlantic jet stream (south, middle, and north). They showed that forecast centers (ECMWF, CMC, and UKMO) exhibited consistent flow-dependent predictability; predictive skill is greatest when the jet is in the south state, linked to greater persistence of that state. Ensemble forecasts diverge most rapidly passing through the north jet state. The sensitivity to initial conditions is associated in this case with Rossby wave breaking and split jet formation.

Patterns associated with persistent behavior have a major influence on regional weather extremes and their impacts. Matsueda (2011) used TIGGE to investigate the predictability of surface temperature in Eurasian blocking events such as the Russian heat wave of 2010 (Dole et al. 2011). While the blocking in June-August of 2010 was predictable on average, even for a lead time of 9 days, there was little skill beyond 6 days in predicting the particular blocking event that brought the severe heat wave (30 July-9 August). Most of the forecasts predicted a decay of the blocking earlier than that observed. At the same time a trough over Pakistan, downstream of the Russian blocking anticyclone, in conjunction with a monsoon depression brought extreme precipitation and flooding to northwest Pakistan (e.g., Galarneau et al. 2012). A key lesson from this case study is that simultaneous extreme events can be linked via Rossby waves but have differing predictability.

Gray et al. (2014) have used TIGGE forecasts to quantify systematic errors in the representation of Rossby waves on the jet stream using diagnostics that were not sensitive to longitudinal phase displacements of waves, namely, the total area occupied by ridges and the average horizontal potential vorticity (PV) gradient across the tropopause. Both ridge area and PV gradient decrease with lead time. None of the models can maintain a gradient as tight as that observed in the face of numerical dissipation, implying that the jet stream is weaker than observed. The decrease in ridge area points to a decline in wave activity in the forecasts. This may be because overly smooth PV gradients resulted in faster dispersion of Rossby wave activity or because incorrect representations of the diabatic processes resulted in a loss of amplitude. Further dynamics research is required to identify the processes responsible for these systematic errors and their consequences for weather events downstream.

Madden-Julian oscillation. The Madden-Julian oscillation (MJO; Madden and Julian 1972) is the dominant mode of intraseasonal variability in the tropics and influences tropical weather and extratropical circulations via large-scale teleconnections. There is only a partial understanding of the dynamics of the MJO and its interaction with convective processes and the surface layers of the ocean, and its prediction remains a major challenge. Although the forecast range of the TIGGE ensembles is shorter than the period of the MJO, the TIGGE data allow a good comparison of the MJO forecasts over about half a cycle of the oscillation.

Matsueda and Endo (2011) assessed the MJO forecast performance of operational medium-range ensemble forecasts by using the TIGGE data for the period of 1 January 2008-31 December 2010 (see the example forecast comparison in Fig. 6). Wheeler and Hendon (2004) defined a bivariate index of the amplitude and phase of the MJO that provides a convenient framework for evaluating the forecasts. Matsueda and Endo (2011) found that ECMWF and the Met Office generally yield the best performances in predicting the MJO; however, they do not always show similar skill. ECMWF performs well in simulating the maintenance and onset of the $\mathrm{MJO}$ in phases 1-4 (where the region of enhanced convection progresses from East Africa across the Indian Ocean and to the Maritime Continent), whereas Met Office and NCEP perform well in phases 5-8 (where the enhanced convection progresses from the Maritime Continent across the Pacific and on to Africa). They also found that simulations of the MJO generally show a slower phase speed and a larger amplitude than that observed. Predicted amplitude over the Maritime Continent (phases 4 and 5), however, tends to be smaller than that observed, suggesting that most models still face the Maritime Continent predictability barrier (Seo et al. 2009). The quasi-real-time MJO forecasts based on TIGGE data are available via the TIGGE museum (see the sidebar on "The TIGGE museum").

Tropical cyclones. Tropical cyclones (TCs) are one of the most destructive atmospheric disturbances on 


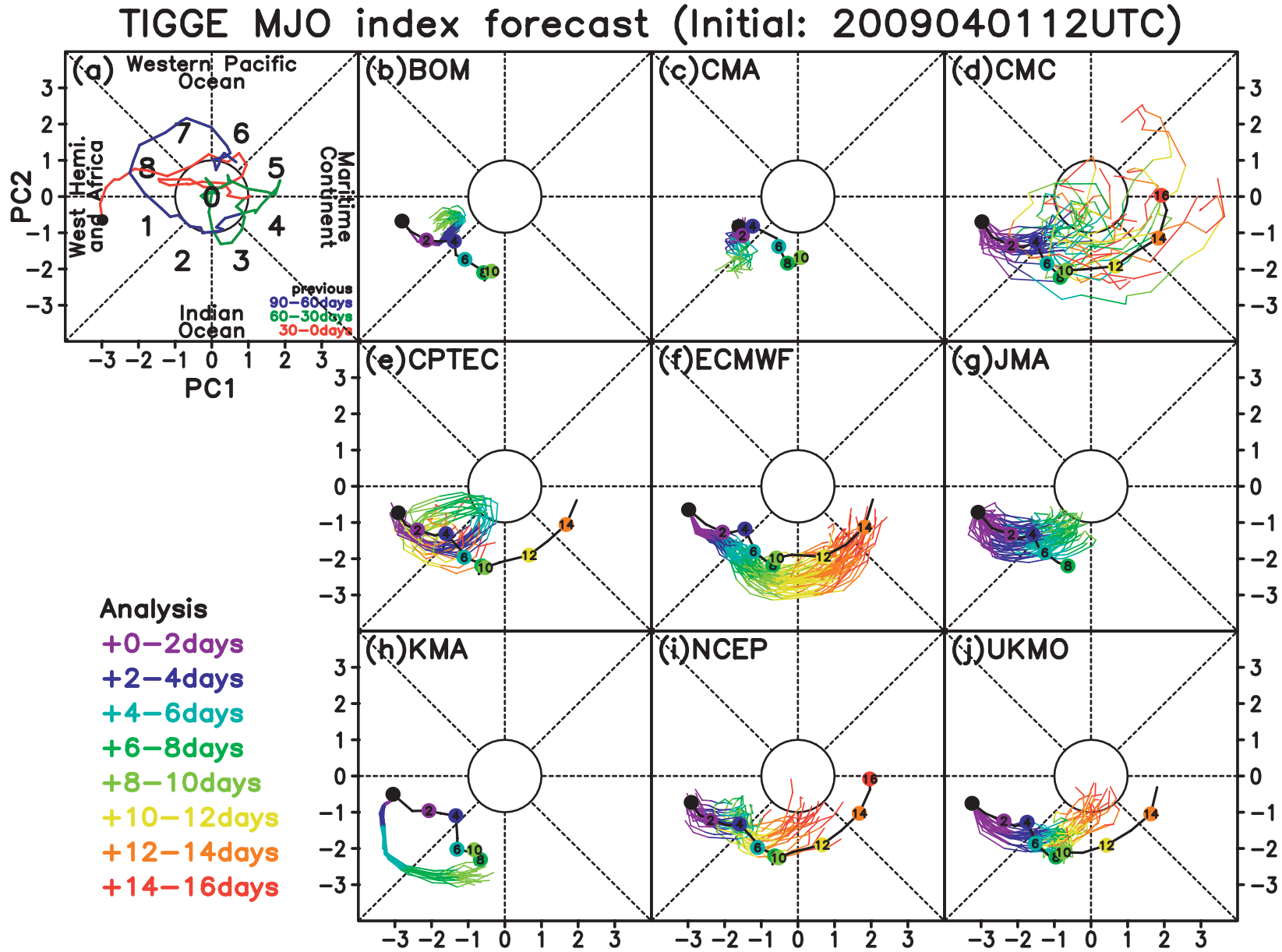

FIG. 6. (a) ECMWF analysis for the Real-time Multivariate MJO index for the 90 days prior to the initial date of the forecast. Real-time Multivariate MJO index forecasts by (b) BoM, (c) CMA, (d) CMC, (e) CPTEC, (f) ECMWF, (g) JMA, (h) KMA, (i) NCEP, and (j) Met Office, initialized at I200 UTC I Apr 2009. The black circle and black line with numbered circles correspond to each analysis (note that there are considerable differences between some of the analyses). The numbers in the colored circles indicate the number of days from the initial date. The colored lines indicate ensemble members. The color changes reflect the lead time of the forecast. Analyses and forecasts generally travel in a counterclockwise direction. [Figure from Matsueda and Endo (20II).]

Earth and pose the greatest threat to life and property (King et al. 2010). Establishing effective warning systems and strengthening international cooperative frameworks are of fundamental importance for disaster risk reduction of TCs. This need is addressed both by improving the underlying TC predictions (discussed in this section) and by developing new informative forecast products (see the section on the "Applications for the forecast user community").

One of the great benefits of TIGGE is that it is now feasible to create and evaluate a multimodel grand ensemble of TC predictions (e.g., Majumdar and Finocchio 2010; Yamaguchi et al. 2012; Matsueda and Nakazawa 2014). Yamaguchi et al. (2012) demonstrated the objective statistical benefits of track forecasts based on a multimodel grand ensemble compared to a single-model ensemble for the western North Pacific basin. However, Majumdar and Finocchio (2010) pointed out that there are some circumstances where combination of ensembles does not improve track forecast skill. On most occasions the observed track should be well within the spread of forecast tracks, but, as shown in Fig. 7, there will be some occasions when the actual track falls on the edge of the forecast ensemble.

It is sometimes necessary to forecast the most likely TC track; in general this will be given by the ensemble-mean track, but Qi et al. (2014) and Tsai and Elsberry (2013) have developed some more sophisticated approaches. Tsai and Elsberry (2013) showed that, in situations where there was a track bifurcation (two clusters of forecast tracks), the track cluster with a percentage greater than $70 \%$ can be reliably selected as the better choice. For situations 
when later observations are available, Qi et al. (2014) developed an approach by which larger weight is given to ensemble members that are closer to the observed TC locations.

For probabilistic predictions of TCs, it is important that the ensemble initial perturbations are a realistic representation of the uncertainties in the initial conditions. TIGGE has helped analyze and interpret the initial perturbations and their impact on TC forecasts (e.g., Hamill et al. 2011; Magnusson et al. 2014). Yamaguchi and Majumdar (2010) demonstrated that singular, vector-based perturbations grow through a baroclinic energy conversion in a vortex, which amplifies the ensemble spread of TC tracks. TIGGE has also contributed to the analysis of the sensitivity of forecasts to initial condition perturbations, which can be used for the targeting of observations to improve TC forecasts (e.g., Majumdar et al. 2011).

TIGGE has facilitated studies on understanding TC dynamics and their prediction across TC basins worldwide. Majumdar and Torn (2014) showed that ensembles have potential for probabilistic prediction out to 5 days. Although the reliable prediction of TC formation is in its infancy, studies using TIGGE data demonstrate skill in predicting formation using multimodel grand ensembles (e.g., Belanger et al. 2012; Halperin et al. 2013). Given that TC intensity changes and genesis events are often affected by environmental influences such as wind vertical shear and tropical waves (e.g., Kepert 2010; Tory and Frank 2010), even relatively low-resolution ensemble data could be beneficial.
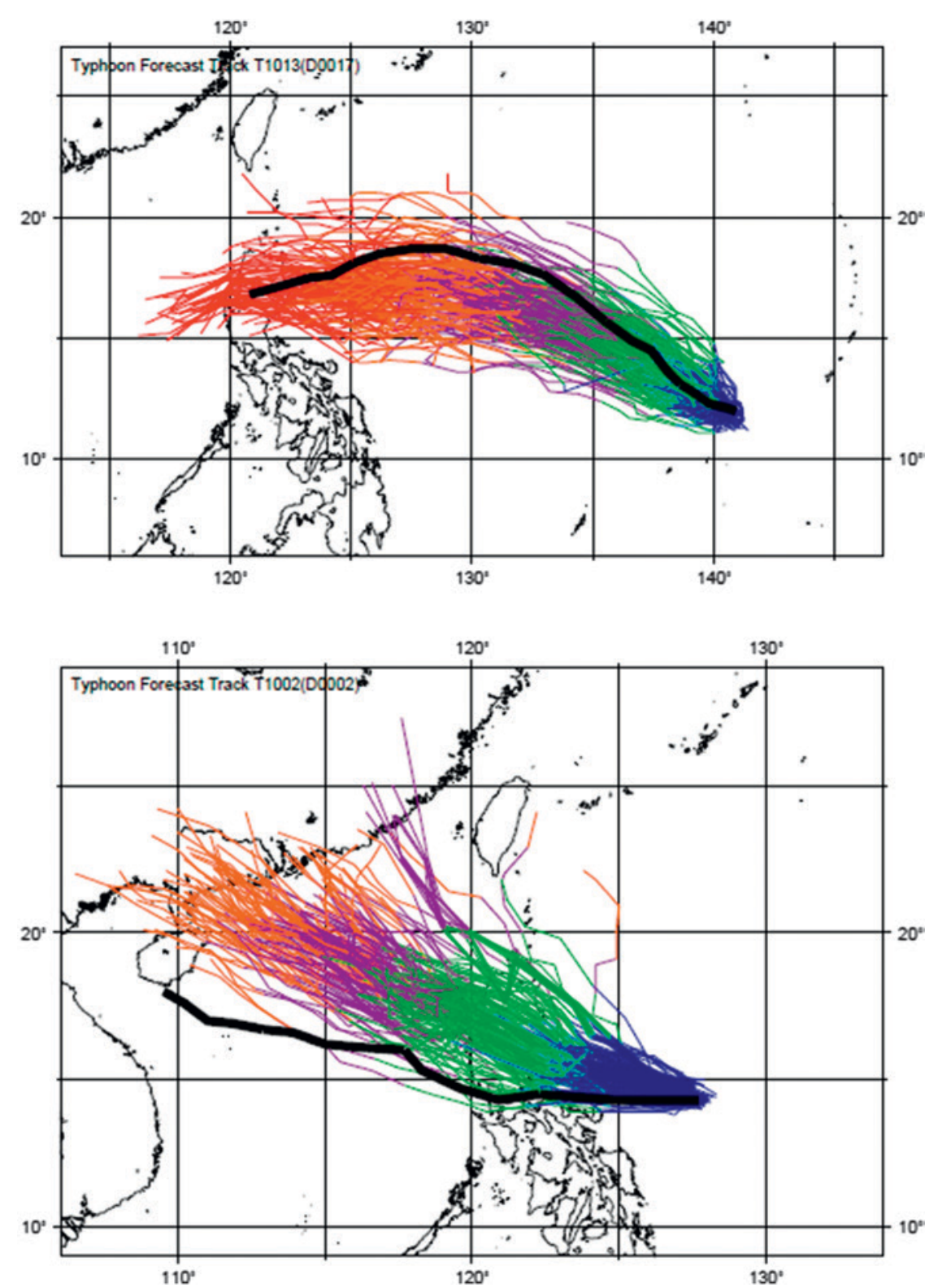

FIG. 7. Track predictions (thin lines) by multimodel grand ensemble (top) for Typhoon Megi initiated at 1200 UTC 25 Oct 2010 and (bottom) for Typhoon Conson initiated at 1200 UTC 12 Jul 2010. The black line is the observed track, and blue, green, purple, orange, and red denote prediction times of I-5 days, respectively (after Yamaguchi et al. 20I2).

Extratropical transition of tropical cyclones. TCs can also have a profound effect on the synoptic evolution in midlatitudes. A poleward moving TC interacts with the midlatitude Rossby waveguide and may undergo extratropical transition (ET), transforming from a tropical into an extratropical cyclone (Jones et al. 2003). The outflow and circulation of the TC may amplify or even trigger the development of a midlatitude Rossby wave train, leading to the potential for high-impact weather in regions downstream 
of the TC itself. The difficulties in representing ET often lead to a decrease in forecast skill, which can be investigated using ensemble forecasts, as demonstrated by Harr et al. (2008), Anwender et al. (2008), and Harr (2010).

TIGGE has opened up the possibility of using a range of ensembles to address the impact of transitioning TCs on predictability in downstream regions. Keller et al. (2011) showed that TIGGE offers a broader range of possible forecast scenarios for ET events and the downstream impact than an ensemble generated by a single forecasting system. Whether these additional scenarios provide a reasonable representation of the uncertainty of the actual development requires further investigation. In a dynamical study using TIGGE data, Archambault et al. (2014) investigated the role of transitioning TC Malakas on the amplification of a midlatitude wave train and the consequent high-impact weather over North America. Both studies highlight the use of TIGGE to further advance our knowledge of ET events and their impact on predictability.

\section{APPLICATIONS FOR THE FORECAST} USER COMMUNITY. Tropical cyclone forecasting. During the THORPEX Pacific Asian Regional Campaign (T-PARC), several TIGGE partners started to exchange tropical cyclone track predictions in near-real time, using an extensible markup language (XML)-based format that was developed for the purpose (cyclone XML or CXML format; see www .bom.gov.au/cyclone/cxmlinfo/). Ensemble forecast products based on the CXML data proved invaluable for the North Western Pacific Tropical Cyclone Ensemble Forecast Project (NWP-TCEFP) that was launched in 2009. During TCEFP, the ensembles were utilized by forecasters from the Economic and Social Commission for Asia and the Pacific (ESCAP)/ WMO Typhoon Committee and also the Southeast Asia region of the WMO Severe Weather Forecast Demonstration Project (SWFDP; see the section on "Early warning products" below). Surveys carried out in conjunction with TCEFP confirmed the usefulness of ensemble TC forecast products for operational forecasting (Yamaguchi et al. 2014).

Although TC track predictions have become significantly more accurate over the past few decades, there is room for improvement in quantifying and communicating uncertainty in the forecasts (e.g., Heming and Goerss 2010). As discussed in the section on "Tropical cyclones," multimodel grand ensembles generally give objectively more skillful forecasts than single-model ensembles. These new TC products provide forecasters with additional information by summarizing the forecast uncertainty from the grand ensemble and so increase the level of confidence in the forecasts.

Some examples of multimodel ensemble products are shown for the forecasts of Hurricane Sandy in Fig. 8. Sandy developed in the Caribbean Sea and was declared a hurricane on 24 October 2012. During its lifetime, Sandy underwent a complex evolution, making landfall in Jamaica, Cuba, and the Bahamas. After tracking over the Atlantic, Sandy turned westward and made landfall unusually far north, near Atlantic City, New Jersey, at 0000 UTC 30 October 2012, with sustained winds of $80 \mathrm{mi} \mathrm{h}^{-1}\left(35.8 \mathrm{~m} \mathrm{~s}^{-1}\right)$ and a central pressure of $945 \mathrm{hPa}$. Because of its huge size, Sandy caused a storm surge along the entire East Coast but particularly in New York and New Jersey, leading to around $\$ 50$ billion (U.S. dollars) in damage and at least 147 fatalities. The National Hurricane Center (NHC) produced a comprehensive report on Sandy and its impact (Blake et al. 2013), while Magnusson et al. (2014) investigated the skill of medium-range forecasts of Sandy.

Figure 8 shows 5-day forecasts of strike probability, individual track, and ensemble-mean track based on three ensembles (ECMWF, NCEP, and Met Office), giving an early warning of the landfall. These plots are produced from 96 equally weighted ensemble members. In this case, the actual track of the storm sits within the areas of highest probability in the strike probability. The ensemble-mean tracks (right-hand side) are plotted for each individual center and the consensus of the three centers.

Early warning products. Using TIGGE data, Matsueda and Nakazawa (2014) have developed a prototype suite of ensemble-based early warning products for severe weather events, using both single-model (ECMWF, JMA, NCEP, and Met Office) and multimodel grand ensembles. These products estimate the forecast probability of the occurrence of heavy rainfall, strong winds, and severe high and low temperatures based on each model's climatology, that is, using information from the climatological probability density function to determine appropriate thresholds for severe weather events. The products are now routinely available as part of the TIGGE Museum.

Objective verification of these products demonstrates that the construction of multimodel grand ensembles by combining four single-model ensembles can improve the skill of probabilistic forecasts of severe events (Matsueda and Nakazawa 2014). The grand ensemble provides more reliable forecasts than 
a) Ensemble forecast tracks

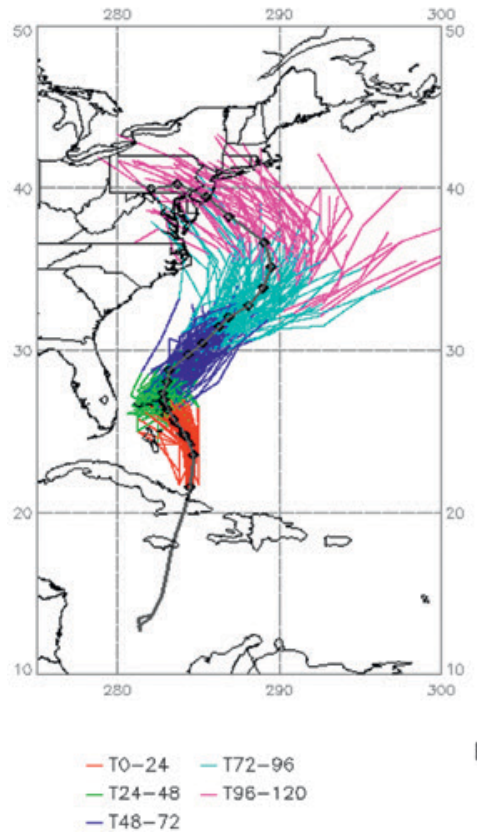

b) Strike probabilities

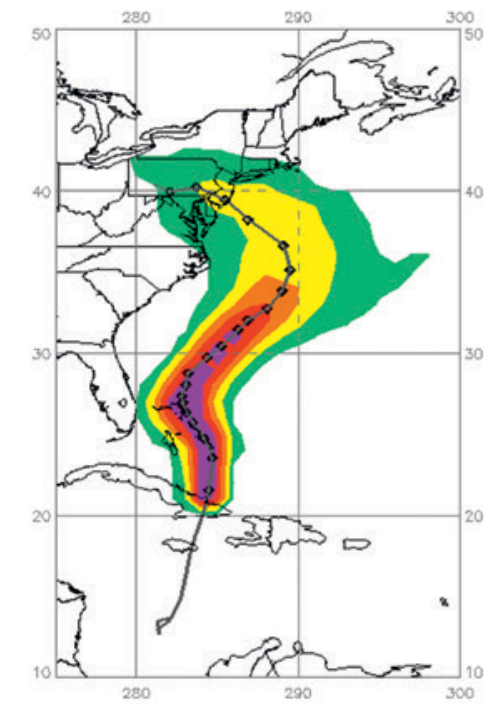

Probability the storm will pass within 75 miles

5-19x 20-39\% $40-59 \%$ 60-79\% $80-100 x$ c) Ensemble mean forecast tracks

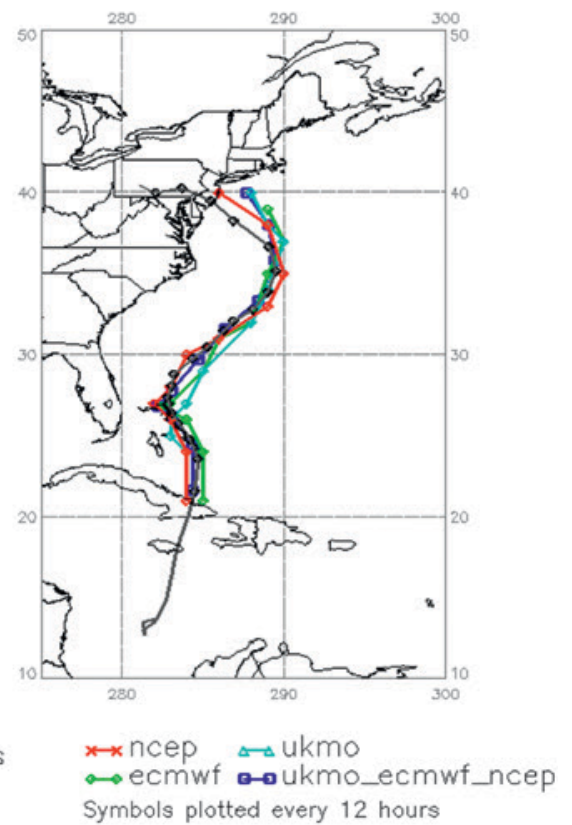

FIG. 8. The 5-day forecasts of (a) individual ensemble tracks, (b) strike probability, and (c) ensemble-mean track forecasts for Hurricane Sandy from I200 UTC 25 Oct 20I2. The strike probability is the probability that the center of the storm will pass within $75 \mathrm{mi}$ (approximately $120 \mathrm{~km}$ ) during the forecast period. The observed track is indicated by a thicker black line with diamond symbols in 6-hourly increments and with a gray line before the forecast period.

single-model ensembles for all lead times, although the grand ensemble is still overconfident, especially for lead times greater than $216 \mathrm{~h}$.

An example of this type of forecast product is shown in Fig. 9 for a heavy precipitation event in West Africa on 1 September 2009 that caused severe flooding in Ouagadougou, Burkina Faso. In all ensembles, there is an indication of the risk of heavy rainfall over West Africa 4-5 days ahead of the event. However, the location of the peak rainfall in Burkina Faso was captured only 2-3 days ahead (not shown). Mesoscale convective systems (MCSs), which lead to such events, are not well predicted by the current ensemble systems. The multimodel ensemble produces a smoother probability map, suggesting that the main benefit of combination for this region is achieved by increasing the ensemble size. Using TIGGE data, Hopsch et al. (2014) showed that the link between large-scale circulation and MCSs could potentially be exploited to improve their prediction.

Since the skill of these TIGGE forecast products has been demonstrated, there is a strong incentive to implement them in real time, avoiding the 2-day delay in accessing data from the TIGGE archive. A system is currently being set up to supply these early warning products to the WMO SWFDP forecasters in real time.
The SWFDP (www.wmo.int/pages/prog/www $/ s w f d p /)$ enables countries in some of the less developed regions of the world to benefit from stateof-the-art numerical model predictions. The global NWP centers generate graphical products that are tailored to support regional SWFDP initiatives. The current SWFDP products will be supplemented both by the ensemble-based early warning products and the multimodel tropical cyclone products developed using TIGGE. Designated regional forecast centers disseminate these products, and associated forecast guidance, to neighboring national meteorological services. The first region to be covered by SWFDP was southern Africa, with Pretoria, South Africa, as the primary regional center; the project has since been extended to cover the South Pacific islands and, more recently, East Africa and Southeast Asia

\section{DISCUSSION AND FUTURE PROSPECTS.}

The TIGGE project has provided a valuable dataset to facilitate research on ensemble techniques, including demonstrating the benefit of combining predictions from several EPSs-this conclusion also carries through to hydrological applications (see sidebar on "Hydrological forecasting"). Although combination has proved a pragmatic approach to improving 


\section{Occurrence probability of extreme $24 \mathrm{hr}$ precipitation Valid: 2009.08.27.12UTC +4-5days}

(a)Grand ens. mem:147

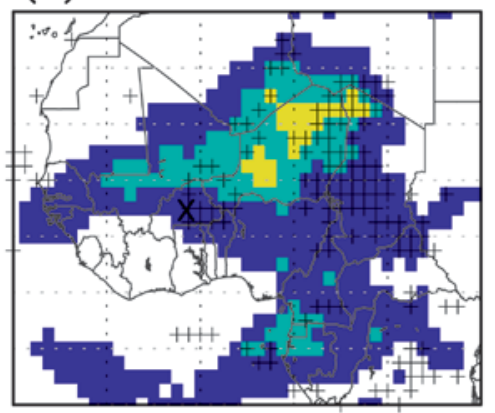

(d)NCEP EPS mem:21

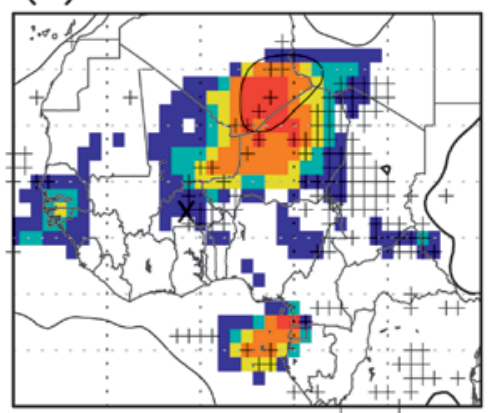

(b)ECMWF EPS mem:51

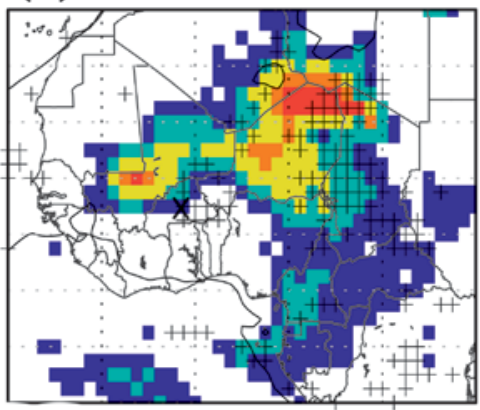

(e)UKMO EPS mem:24

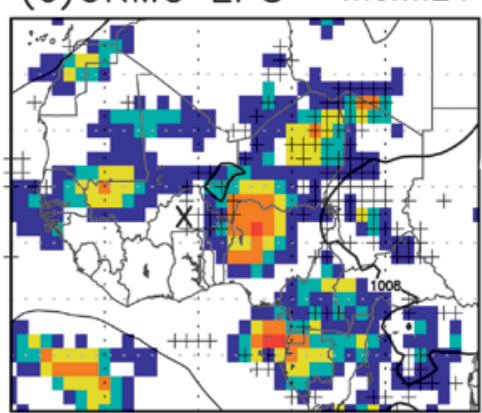

contour: control SLP [hPa]
+ : extremes observed $\left(90^{\text {th }}\right)$

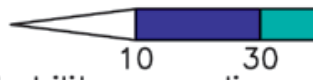

50

70

90

probability exceeding climatological $90^{\text {th }}$ percentile [\%]

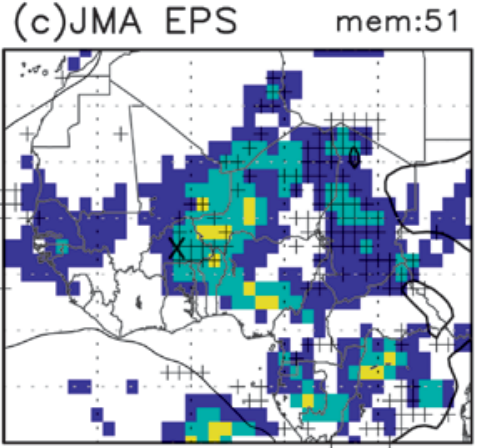

(f)Extremes observed

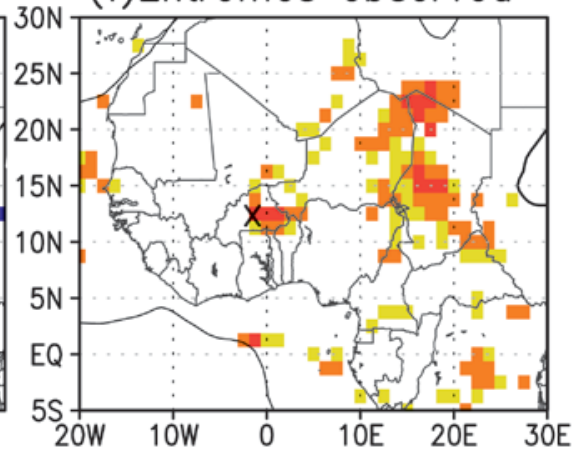

contour: observed SLP [hPa]

observed extremes defined with $90^{\text {th }}, 95^{\text {th }}$ and $99^{\text {th }}$ percentiles

FiG. 9. Occurrence probabilities of heavy rainfall on I Sep 2009, when there was severe flooding in Ouagadougou (marked with an $\mathrm{X}$ ). The shading indicates occurrence probabilities by the (a) multimodel grand ensemble, (b) ECMWF ensemble, (c) JMA ensemble, (d) NCEP ensemble, and (e) Met Office ensemble, initialized at I200 UTC 27 Aug and showing rainfall for I200 UTC 3I Aug to I200 UTC I Sep 2009. Contours in (b)-(e) indicate predicted sea level pressure in each control run. The climatological 90 th percentiles of the models at each lead time were used to define the predicted extremes. (f) Observed rainfall from the Global Satellite Mapping of Prediction (GSMaP) dataset, relative to observed climatology, and observed pressure (contours).

probabilistic forecast skill, we expect less benefit from the technique in the future, as systematic errors in ensembles are reduced. TIGGE has also supported a wide range of research on dynamics, the fundamental nature of predictability, and development of forecast applications.

In view of TIGGE's success, it has been agreed that the project should continue for $5 \mathrm{yr}$ further beyond the completion of the THORPEX research program at the end of 2014. (Any extension beyond 2019 will be considered nearer the time.) The great majority of TIGGE partners will continue to participate and provide ensemble predictions for use by the research community. Both ECMWF and CMA will continue to host TIGGE archive centers. Building on the success of THORPEX, WWRP has established three THORPEX legacy projects: the Subseasonal to Seasonal Prediction (S2S) and Polar Prediction (PPP) projects are already underway, while the High-Impact Weather (HIWeather) project kick-off meeting has been scheduled for April 2016. S2S explores the longer-range prediction problem, when the interactions between the atmosphere and other elements of the Earth system, especially oceans, are increasingly important. S2S is a joint initiative between World Climate Research Program (WCRP) and WWRP. This project is underpinned by the S2S database, which went live during 2015. This S2S database is based closely on the TIGGE database, using similar data formats and conventions, which should facilitate research on seamless predictions ranging from 1 day to 2 months ahead, using both 


\section{HYDROLOGICAL FORECASTING}

ydrological models act as nonlinear filters and integrators of rainfall predictions. They are therefore ideal for understanding the impact of deficiencies in the ensemble forecasts for downstream applications.

TIGGE was first used for hydrometeorological forecasting when Pappenberger et al. (2008) demonstrated the potential of grand ensembles for early flood warning, applying the European Flood Awareness System (EFAS, Thielen et al. 2009) to a hindcasted flood event in Romania. Figure SBI, from this study, shows forecasts of river level for a point on the river Jiu that was severely flooded in October 2007, based on seven single-model ensembles and a multimodel grand ensemble. While all the ensembles predict the onset of the rising river level correctly, only two single-model ensembles and the multimodel ensemble bracket the flood peak. The conclusion of the study was that if grand ensemble forecasts had been used, flood warnings could have been issued 8 days before the event, whereas warnings based on a single ensemble system would only have allowed for a lead time of 4 days.

Several studies have now shown that a TIGGE-based approach increases lead time and skill across many climatic regions (e.g., Bao and Zhao
2012; Pappenberger et al. 2008). The information gain in applying TIGGE for hydrological forecasts has proven to be consistent in a way that is independent of the hydrological model applied. However, there is a clear sensitivity to catchment size: the smaller the catchment, the more important ensemble postprocessing, calibration, and combination becomes, as shown by $\mathrm{He}$ et al. (2009) for a mesoscale catchment area in the Midlands area of the United Kingdom. It is clear that the TIGGE archive has been of incredible value for furthering research in hydrometeorological forecasting and demonstrating the potential of earlier flood warning.

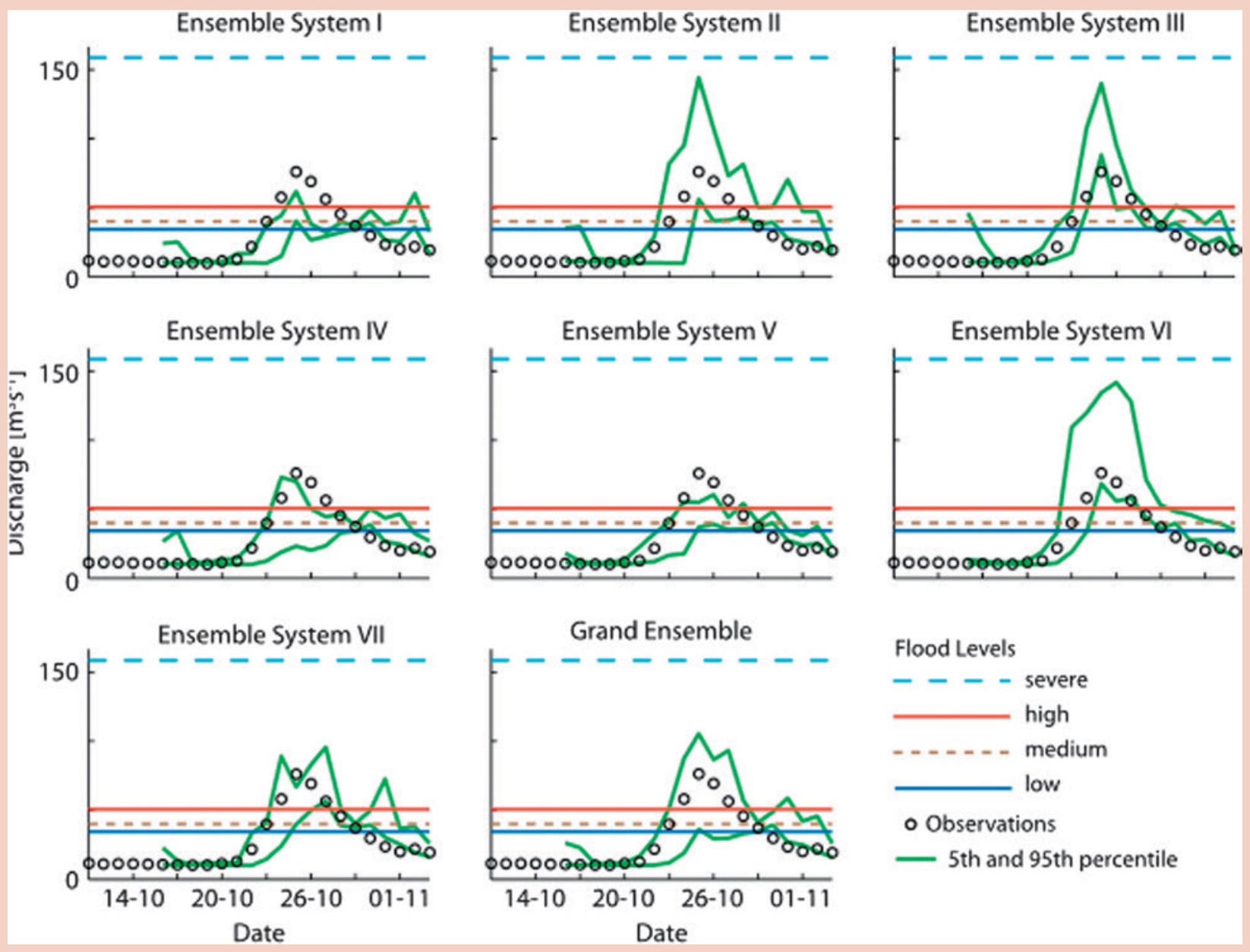

FIG. SBI. River discharge predictions for a point on the river Jiu, Romania, where flooding was observed. The 5th and 95th percentiles of predictions are shown for the different forecasts with a 5-day lead time. The dashed horizontal lines show the four EFAS warning thresholds. "Observed" discharges refer to simulations based on observed meteorological input. [Figure from Pappenberger et al. (2008).] 
datasets. PPP is concerned with the prediction of weather in high latitudes and its link with lower latitudes (e.g., Jung and Matsueda 2015). The main focus of PPP will be preparing for, coordinating, and analyzing results from the Year of Polar Prediction (YOPP), a combined modeling and field campaign that is planned to take place between mid-2017 and mid-2019. TIGGE will play a key role in providing ensemble prediction data for PPP, and some enhancements to TIGGE may be implemented to support the requirements of PPP or other WWRP projects. The HIWeather project addresses the improvement of forecasts and warnings of high-impact weather, with a focus on five hazard areas: urban flooding, localized extreme wind, wildfire, urban heat and air quality, and disruptive winter weather. A key aspect of the project will be understanding vulnerability and risk and improving the communication of warnings of high-impact weather.

Looking forward, increases in computer performance allow short-range convective-scale ensemble forecasts that will be a major step forward to the prediction of details of hazardous weather. Currently both Deutscher Wetterdienst (DWD) and the Met Office are running operational ensemble systems with around a 2-km grid; Météo-France and other centers also have high-resolution systems under development. The U.S. Hazardous Weather Testbed project has been running for more than a decade, comparing experimental ensemble forecasts run at $4-\mathrm{km}$ resolution across the central United States (Clark et al. 2012). The recent establishment of the European TIGGE-LAM archive means that forecasts from high-resolution ensembles will be more readily available to the research community, and it is hoped that similar facilities will, in the future, be developed on other continents. These datasets will provide invaluable data to underpin the focus on improving the detailed prediction of high-impact weather events at short time scales and should prove especially valuable for the HIWeather project.

Ensemble methods are also being increasingly employed in data assimilation in both purely ensemble approaches to data assimilation (e.g., Houtekamer et al. 2005) and hybrid ensemble-variational methods (e.g., Clayton et al. 2013). An ensemble of model states provides a good framework to specify the relationship between uncertainties in model variables; that is, well-specified ensemble perturbations should be closely related to the background error covariance information that is used for data assimilation. A very large ensemble is needed in order to satisfactorily represent the error covariance information in an ensemble data assimilation system, while hybrid techniques permit the use of fewer ensemble members by combining flow-dependent information from an ensemble with static climatological error covariances.

A new WWRP working group on predictability, dynamics, and ensemble forecasting (PDEF) has been established to address the theoretical basis of ensemble forecasting and its relation to the dynamics of the atmospheric phenomena and coupled systems. Research with TIGGE has highlighted some key aspects of flow-dependent predictability on the large scale and connections with high-impact weather events. The new generation of convective-scale ensembles raises many important issues: the suitability of data assimilation approaches developed for the synoptic scale, the construction of ensembles, the role of stochastic parameterization in representing model uncertainty, and the fundamental nature of predictability itself on finer spatial and temporal scales. The PDEF working group will be scientifically responsible for the development of the TIGGE and TIGGE-LAM datasets to promote and support ongoing scientific research and especially the THORPEX legacy projects. The working group will consider enhancements to TIGGE to support future research needs, for example, possible additional variables to support WWRP projects. PDEF will bring dynamical expertise from the academic community to bear on these exciting new challenges, ultimately driving toward improved probabilistic prediction.

In recent years, there has been a rapid growth in the utilization of probabilistic forecasts by both industry and government organizations to manage risks. The TIGGE project has been at the forefront of these developments, making a major contribution to the development of ensemble methods to provide these risk-based forecasts. The multiyear ensemble forecast dataset has been an unparalleled resource to the applied science research community. TIGGE has also provided a rich stream of data that has been used for a range of studies, covering research on atmospheric dynamics, improvement of predictive skill of models, and development of ensemble techniques. Ensemble techniques are increasingly important for prediction at both short space and time scales, extending the limits of predictability and data assimilation. Looking forward, we expect TIGGE and TIGGE-LAM to support a range of exciting developments, underpinning further improvements to the use of ensemble techniques in both data assimilation and prediction and also the developments of a rich collection of risk-based forecasting applications. 
ACKNOWLEDGMENTS. We are indebted to Steve Worley, Doug Schuster, Baudouin Raoult, Manuel Fuentes, and their colleagues for setting up and managing the TIGGE archives at ECMWF, NCAR, and CMA. The TIGGE project would not have been possible without support from the TIGGE partnership: the three archive centers, the 10 data providers, the National Climatic Data Center, and the members of the THORPEX GIFS-TIGGE working group. We are grateful for funding from the European Commission via the GEOWOW project (Grant Agreement 282915), which supported enhancements to the ECMWF TIGGE archive, and some of the development and evaluation of severe weather forecast products.

\section{REFERENCES}

Anwender, D., P. A. Harr, and S. C. Jones, 2008: Predictability associated with the downstream impacts of the extratropical transition of tropical cyclones: Case studies. Mon. Wea. Rev., 136, 3226-3247, doi:10.1175/2008MWR2249.1.

Archambault, H. M., P. A. Harr, and R. W. Moore, 2014: A diagnosis of the poorly forecast interaction between recurving Tropical Cyclone Malakas (2010) and the extratropical flow. 31st Conf. on Hurricanes and Tropical Meteorology, San Diego, CA, Amer. Meteor. Soc., 168. [Available online at https://ams.confex .com/ams/31Hurr/webprogram/Paper245248.html.]

Bao, H., and L. Zhao, 2012: Development and application of an atmospheric-hydrologic-hydraulic flood forecasting model driven by TIGGE ensemble forecasts. Acta Meteor. Sin., 26, 93-102, doi:10.1007/ s13351-012-0109-0.

Belanger, J. I., P. J. Webster, J. A. Curry, and M. T. Jelinek, 2012: Extended prediction of north Indian Ocean tropical cyclones. Wea. Forecasting, 27, 757-769, doi:10.1175/WAF-D-11-00083.1.

Blake, E. S., T. B. Kimberlain, R. J. Berg, J. P. Cangialosi, and J. L. Beven II, 2013: Tropical Cyclone Report Hurricane Sandy. National Hurricane Center Rep. AL182012, 157 pp.

Bougeault, P., and Coauthors, 2010: The THORPEX Interactive Grand Global Ensemble (TIGGE). Bull. Amer. Meteor. Soc., 91, 1059-1072, doi:10.1175/2010BAMS2853.1.

Candille, G., 2009: The multiensemble approach: The NAEFS example. Mon. Wea. Rev., 137, 1655-1665, doi:10.1175/2008MWR2682.1.

Cassou, C., L. Terray, J. W. Hurrell, and C. Deser, 2004: North Atlantic winter climate regimes: Spatial asymmetry, stationarity with time, and oceanic forcing. J. Climate, 17, 1055-1068, doi:10.1175/1520 -0442(2004)017<1055:NAWCRS>2.0.CO;2.
Clark, A. J., and Coauthors, 2012: An overview of the 2010 Hazardous Weather Testbed experimental forecast program spring experiment. Bull. Amer. Meteor. Soc., 93, 55-74, doi:10.1175/BAMS-D-11-00040.1.

Clayton, A. M., A. C. Lorenc, and D. M. Barker, 2013: Operational implementation of a hybrid ensemble/4D-Var global data assimilation system at the Met Office. Quart. J. Roy. Meteor. Soc., 139, 1445-1461, doi:10.1002/qj.2054.

Dawson, A., T. N. Palmer, and S. Corti, 2012: Simulating regime structures in weather and climate prediction models. Geophys. Res. Lett., 39, L21805, doi:10.1029/2011GL049901.

Dole, R., and Coauthors, 2011: Was there a basis for anticipating the 2010 Russian heat wave? Geophys. Res. Lett., 38, L06702, doi:10.1029/2010GL046582.

Frame, T. H. A., M. H. P. Ambaum, S. L. Gray, and J. Methven, 2011: Ensemble prediction of transitions of the North Atlantic eddy-driven jet. Quart. J. Roy. Meteor. Soc., 137, 1288-1297, doi:10.1002/qj.829.

_ - J. Methven, S. L. Gray, and M. H. P. Ambaum, 2013: Flow-dependent predictability of the North Atlantic jet. Geophys. Res. Lett., 40, 2411-2416, doi:10.1002 /grl.50454.

Froude, L. S. R., 2010: TIGGE: Comparison of the prediction of Northern Hemisphere extratropical cyclones by different ensemble prediction systems. Wea. Forecasting, 25, 819-836, doi:10.1175/2010WAF2222326.1.

_ , 2011: TIGGE: Comparison of the prediction of Southern Hemisphere extratropical cyclones by different ensemble prediction systems. Wea. Forecasting, 26, 388-398, doi:10.1175/2010WAF2222457.1.

Galarneau, T. J., T. M. Hamill, R. M. Dole, and J. Perlwitz, 2012: A multiscale analysis of the extreme weather events over western Russia and northern Pakistan during July 2010. Mon. Wea. Rev., 140, 1639-1664, doi:10.1175/MWR-D-11-00191.1.

Gray, S. L., C. Dunning, J. Methven, G. Masato, and J. Chagnon, 2014: Systematic model forecast error in Rossby wave structure. Geophys. Res. Lett., 41, 2979-2987, doi:10.1002/2014GL059282.

Hagedorn, R., F. J. Doblas-Reyes, and T. N. Palmer, 2005: The rationale behind the success of multimodel ensembles in seasonal forecasting. Part I: Basic concept. Tellus, 57A, 219-233, doi:10.1111/j.1600 -0870.2005.00103.x.

— R. Buizza, T. M. Hamill, M. Leutbecher, and T. N. Palmer, 2012: Comparing TIGGE multi-model forecasts with reforecast-calibrated ECMWF ensemble forecasts. Quart. J. Roy. Meteor. Soc., 138, 1814-1827, doi:10.1002/qj.1895.

Halperin, D. J., H. E. Fuelberg, R. E. Hart, J. H. Cossuth, P. Sura, and R. J. Pasch, 2013: An evaluation of 
tropical cyclone genesis forecasts from global numerical models. Wea. Forecasting, 28, 1423-1445, doi:10.1175/WAF-D-13-00008.1.

Hamill, T. M., 2012: Verification of TIGGE multimodel and ECMWF reforecast-calibrated probabilistic precipitation forecasts over the contiguous United States. Mon. Wea. Rev., 140, 2232-2252, doi:10.1175 /MWR-D-11-00220.1.

—, J. S. Whitaker, M. Fiorino, and S. G. Benjamin, 2011: Global ensemble predictions of 2009's tropical cyclones initialized with an ensemble Kalman filter. Mon. Wea. Rev., 139, 668-688, doi:10.1175/2010MWR3456.1.

Harr, P. A., 2010: The extratropical transition of tropical cyclones: Structural characteristics, downstream impacts, and forecast challenges. Global Perspectives on Tropical Cyclones, J. C. L. Chan and J. D. Kepert, Ed., World Scientific, 149-174.

—, D. Anwender, and S. C. Jones, 2008: Predictability associated with the downstream impacts of the extratropical transition of tropical cyclones: Methodology and a case study of Typhoon Nabi (2005). Mon. Wea. Rev., 136, 3205-3225, doi:10.1175 /2008MWR2248.1.

He, Y., F. Wetterhall, H. L. Cloke, F. Pappenberger, M. Wilson, J. Freer, and G. McGregor, 2009: Tracking the uncertainty in flood alerts driven by grand ensemble weather predictions. Meteor. Appl., 16, 91-101, doi:10.1002/met.132.

Heming, J., and J. Goerss, 2010: Track and structure forecasts of tropical cyclones. Global Perspectives on Tropical Cyclones, C. L. Chan and J. D. Kepert, Ed., World Scientific, 287-323.

Hewson, T. D., and H. A. Titley, 2010: Objective identification, typing and tracking of the complete life-cycles of cyclonic features at high spatial resolution. Meteor. Appl., 17, 355-381, doi:10.1002/met.204.

— , L. Magnusson, O. Breivik, F. Prates, I. Tsonevsky, and J. W. de Vries, 2014: Windstorms in northwest Europe in late 2013. ECMWF Newsletter, No. 139, ECMWF, Reading, United Kingdom, 122-128.

Hopsch, S., S. C. Jones, and J. H. Keller, 2014: Analysis of tropical high impact weather events using TIGGE data. 31st Conf. on Hurricanes and Tropical Meteorology, San Diego, CA, Amer. Meteor. Soc., 2B.3. [Available online at https://ams.confex.com/ams/31Hurr /webprogram/Paper244638.html.]

Houtekamer, P. L., H. L. Mitchell, G. Pellerin, M. Buehner, M. Charron, L. Spacek, and B. Hansen, 2005: Atmospheric data assimilation with an ensemble Kalman filter: Results with real observations. Mon. Wea. Rev., 133, 604-620, doi:10.1175 /MWR-2864.1.
Johnson, C., and R. Swinbank, 2009: Medium-range multimodel ensemble combination and calibration. Quart. J. Roy. Meteor. Soc., 135, 777-794, doi:10.1002 /qj.383.

Jolliffe, I. T., and D. B. Stephenson, 2011: Forecast Verification: A Practitioner's Guide in Atmospheric Science. 2nd ed. Wiley and Sons, $274 \mathrm{pp}$.

Jones, S. C., and Coauthors, 2003: The extratropical transition of tropical cyclones: Forecast challenges, current understanding, and future directions. Wea. Forecasting, 18, 1052-1092, doi:10.1175/1520 -0434(2003)018<1052:TETOTC>2.0.CO;2.

Jung, T., and M. Matsueda, 2015: Verification of global numerical weather forecasting systems in polar regions using TIGGE data. Quart. J. Roy. Meteor. Soc., doi:10.1002/qj.2437, in press.

Keller, J. H., S. C. Jones, J. L. Evans, and P. A. Harr, 2011: Characteristics of the TIGGE multimodel ensemble prediction system in representing forecast variability associated with extratropical transition. Geophys. Res. Lett., 38, L12802, doi:10.1029/2011GL04727.

Kepert, J. D., 2010: Tropical cyclone structure and dynamics. Global Perspectives on Tropical Cyclones, J. C. L. Chan and J. D. Kepert, Ed., World Scientific, 3-53.

King, D., J. Davidson, and L. Anderson-Berry, 2010: Disaster mitigation and societal impacts. Global Perspectives on Tropical Cyclones, J. C. L. Chan and J. D. Kepert, Ed., World Scientific, 409-436.

Madden, R. A., and P. R. Julian, 1972: Description of global-scale circulation cells in the tropics with a 40-50 day period. J. Atmos. Sci., 29, 1109-1123, doi:10.1175/1520 -0469(1972)029<1109:DOGSCC>2.0.CO;2.

Magnusson, L., A. Thorpe, M. Bonavita, S. Lang, T. McNally, N. Wedi, and M. Yamaguchi, 2014: Evaluation of forecasts for Hurricane Sandy. Mon. Wea. Rev., 142, 1962-1981, doi:10.1175/MWR-D-13 -00228.1.

Majumdar, S. J., and P. M. Finocchio, 2010: On the ability of global ensemble prediction systems to predict tropical cyclone track probabilities. Wea. Forecasting, 25, 659-680, doi:10.1175/2009WAF2222327.1.

— , and R. D. Torn, 2014: Probabilistic verification of global and mesoscale ensemble forecasts of tropical cyclogenesis. Wea. Forecasting, 29, 1181-1198, doi:10.1175/WAF-D-14-00028.1.

_- S.-G. Chen, and C.-C. Wu, 2011: Characteristics of ensemble transform Kalman filter adaptive sampling guidance for tropical cyclones. Quart. J. Roy. Meteor. Soc., 137, 503-520, doi:10.1002/qj.746.

Matsueda, M., 2009: Blocking predictability in operational medium-range ensemble forecasts. SOLA, 5, 113-116, doi:10.2151/sola.2009-029. 
- 2011: Predictability of Euro-Russian blocking in summer of 2010. Geophys. Res. Lett., 38, L06801, doi:10.1029/2010GL04655.

—_, and H. L. Tanaka, 2008: Can MCGE outperform the ECMWF ensemble? SOLA, 4, 77-80, doi:10.2151 /sola.2008-020.

_- and H. Endo, 2011: Verification of medium-range MJO forecasts with TIGGE. Geophys. Res. Lett., 38, L11801, doi:10.1029/2011GL047480.

_ , and T. Nakazawa, 2014: Early warning products for severe weather events derived from operational medium-range ensemble forecasts. Meteor. Appl., 22, 213-222, doi:10.1002/met.1444.

Pappenberger, F., J. Bartholmes, J. Thielen, H. L. Cloke, R. Buizza, and A. de Roo, 2008: New dimensions in early flood warning across the globe using grandensemble weather predictions. Geophys. Res. Lett., 35, L10404, doi:10.1029/2008GL033837.

Park, Y.-Y., R. Buizza, and M. Leutbecher, 2008: TIGGE: Preliminary results on comparing and combining ensembles. Quart. J. Roy. Meteor. Soc., 134, 20292050, doi:10.1002/qj.334.

Qi, L., H. Yu, and P. Chen, 2014: Selective ensemblemean technique for tropical cyclone track forecast by using ensemble prediction systems. Quart. J. Roy. Meteor. Soc., 140, 805-813, doi:10.1002/qj.2196.

Richardson, D., R. Buizza, and R. Hagedorn, 2005: Final report on the first workshop on the THORPEX Interactive Grand Global Ensemble (TIGGE). World Meteorological Organization Rep. WMO/TD-No. 1273, 39 pp.

Seo, K.-H., W. Wang, J. Gottschalck, Q. Zhang, J.-K. E. Schemm, W. R. Higgins, and A. Kuma, 2009: Evaluation of MJO forecast skill from several statistical and dynamical forecast models. J. Climate, 22, 2372-2388, doi:10.1175/2008JCLI2421.1.

Shapiro, M., and A. Thorpe, 2004a: THORPEX: A global atmospheric research programme for the beginning of the 21st century. WMO Bull., 53, 222-226.

$\longrightarrow$, and — , 2004b: THORPEX international science plan. World Meteorological Organization Rep. WMO/TD-No. 1246, 57 pp. [Available online at www.wmo.int/pages/prog/arep/wwrp/new /documents/CD_ROM_international_science_plan _v3.pdf.]
Thielen, J., J. Bartholmes, M.-H. Ramos, and A. de Roo, 2009: The European Flood Alert System-Part 1: Concept and development. Hydrol. Earth Syst. Sci., 13, 125-140, doi:10.5194/hess-13-125-2009.

Tory, K. J., and W. M. Frank, 2010: Tropical cyclone formation. Global Perspectives on Tropical Cyclones, J. C. L. Chan and J. D. Kepert, Ed., World Scientific, 55-91.

Tsai, H.-C., and R. L. Elsberry, 2013: Detection of tropical cyclone track changes from the ECMWF ensemble prediction system. Geophys. Res. Lett., 40, 797-801, doi:10.1002/grl.50172.

Wheeler, M. C., and H. H. Hendon, 2004: An allseason real-time multivariate MJO index: Development of an index for monitoring and prediction. Mon. Wea. Rev., 132, 1917-1932, doi:10.1175/1520 -0493(2004)132<1917:AARMMI>2.0.CO;2.

Wilks, D. S., 2006: Statistical Methods in the Atmospheric Sciences. 2nd ed. Academic Press, 627 pp.

Wilson, L. J., S. Beauregard, A. E. Raftery, and R. Verret, 2007: Calibrated surface temperature forecasts from the Canadian ensemble prediction system using Bayesian model averaging. Mon. Wea. Rev., 135 1364-1385, doi:10.1175/MWR3347.1.

WMO, 2005: THORPEX international research implementation plan. World Meteorological Organization Rep. WMO/TD-No. 1258, 95 pp. [Available online at www.wmo.int/pages/prog/arep/wwrp/new/docu ments/CD_ROM_implementation_plan_v1.pdf.]

Worley, S., D. Schuster, P. Bougeault, B. Raoult, D. H. Chen, and J. Dong, 2008: Improving high-impact weather forecasts. Eos, Trans. Amer. Geophys. Union, 89, 330-331, doi:10.1029/2008EO360002.

Yamaguchi, M., and S. J. Majumdar, 2010: Using TIGGE data to diagnose initial perturbations and their growth for tropical cyclone ensemble forecasts. Mon. Wea. Rev., 138, 3634-3655, doi:10.1175/2010MWR3176.1.

_ - T. Nakazawa, and S. Hoshino, 2012: On the relative benefits of a multi-centre grand ensemble for tropical cyclone track prediction in the western North Pacific. Quart. J. Roy. Meteor. Soc., 138, 2019-2029, doi:10.1002/qj.1937.

,-- , and ——, 2014: North western Pacific tropical cyclone ensemble forecast project. Trop. Cyclone Res. Rev., 3, 193-201, doi:10.6057/2014TCRR03.05. 